\title{
Photospheric magnetic field of an eroded-by-solar-wind coronal mass ejection
}

\author{
J. Palacios, C. Cid, E. Saiz and A. Guerrero \\ Departamento de Física y Matemáticas, \\ Universidad de Alcalá \\ University Campus, Sciences Building, P.O. 28871, Alcalá de Henares, Spain \\ email: judith.palacios@uah.es
}

\begin{abstract}
We have investigated the case of a coronal mass ejection that was eroded by the fast wind of a coronal hole in the interplanetary medium. When a solar ejection takes place close to a coronal hole, the flux rope magnetic topology of the coronal mass ejection (CME) may become misshapen at $1 \mathrm{AU}$ as a result of the interaction. Detailed analysis of this event reveals erosion of the interplanetary coronal mass ejection (ICME) magnetic field. In this communication, we study the photospheric magnetic roots of the coronal hole and the coronal mass ejection area with HMI/SDO magnetograms to define their magnetic characteristics.
\end{abstract}

Keywords. Sun: coronal mass ejections (CMEs), Sun: magnetic fields, (Sun:) solar wind, (Sun:) solar-terrestrial relations

\section{Introduction}

The interaction between solar wind and magnetic structures as ICMEs, are subject of extensive research. These interactions in the interplanetary medium may consist of pileup events (Kataoka et al. 2015), in deflections with coronal holes or with another ICME, as in Lugaz et al.(2011), Lugaz et al.(2012), among other phenomena. The interaction of interest in this work is the magnetic erosion (e.g., Dasso et al. 2006), that is, the magnetic reconnection that can take place in a side or a part of a magnetic cloud due to the mainly unipolar polarity of the solar wind. This fact has important implications, since when the magnetic cloud becomes eroded, it loses part of the typical geometry and may lead to misclassifications of the magnetic cloud axis and orientation, and therefore, to an underconsideration of its actual geoeffectiveness when a 'robust' flux rope model is propagated up to $1 \mathrm{AU}$. Therefore, this kind of cases are relevant for the estimation of how distorted the magnetic cloud may be. This fact has been statistically and numerically studied in Lavraud et al.(2014), Ruffenach et al.(2015).

In the paper of Cid et al.(2016) we describe thoroughly the event. The occurrence was a faint CME ejected in 2015 January 3, that left a dimming close to a boundary of the southern pole coronal hole. Four days later, a moderate geomagnetic storm at ground was registered.

In Cid et al.(2016) we discarded many of the possible sources of the CME, finally concluding that the CME is peeled off from a location that is very close to the northeasternmost rim of the coronal hole. This location suffered an important dimming (transient reduced density area in the corona) from 2015 January 3 00:00 UT to January 3 12:00 UT. The CME, albeit faint, was easy to track. However, we did not find a chromospheric counterpart of the ejection, as a clear filament eruption. Therefore, the hypothesis of a flux rope emerging and erupting could not be validated. In this work we aim at studying the photospheric magnetic field to investigate this hypothesis. 


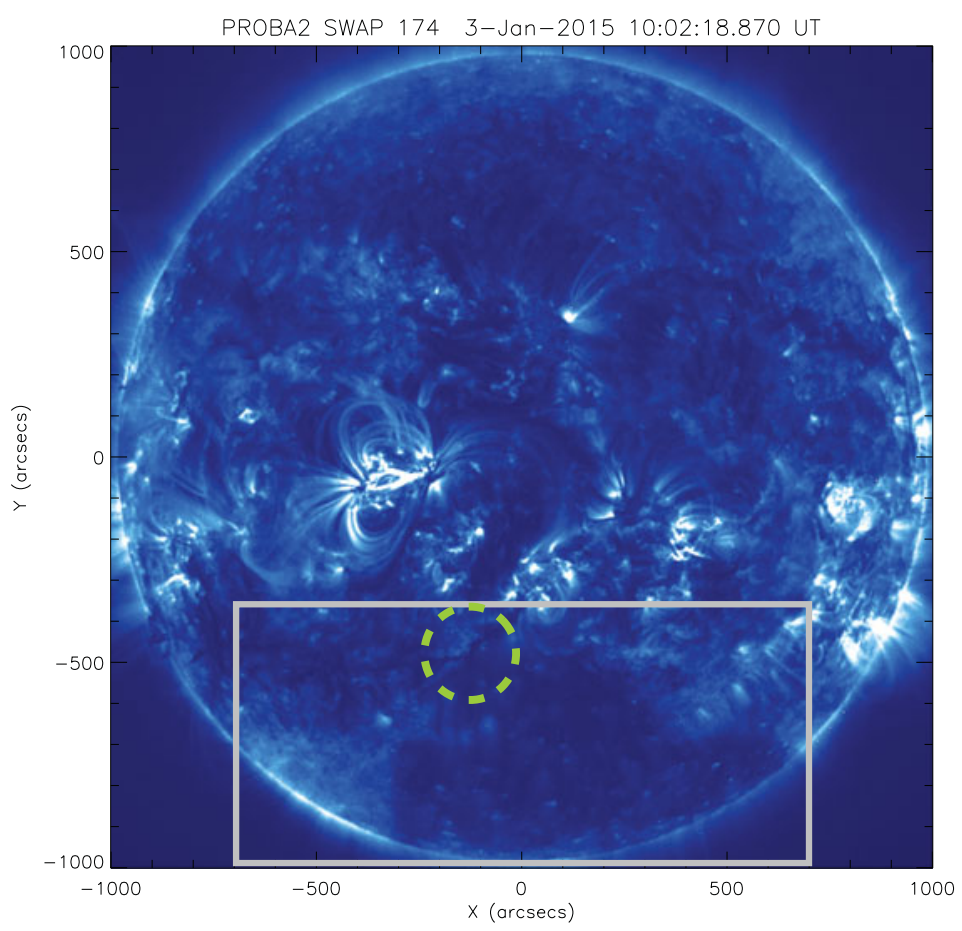

Figure 1. Proba2-SWAP enhanced feature image. The (gray) box encloses the whole southern pole $\mathrm{CH}$, and the (green) dashed circumference highlights the dimming location.

For an illustrative purpose, we have used a Proba2-SWAP image as a context image to show the solar location of the eruptive event. The image has been filtered to enhance spatial high-frequency features, mainly the $\mathrm{CH}$ boundary. The location of the large coronal hole is enclosed by a gray box, while the dimming location is highlighted with a dashed (green) circumference at Figure 1.

\section{Data and results}

To check whether there is any fundamental photospheric magnetic change due to the CME eruption and dimming opening due to the plausible emergence of a flux rope, we computed the photospheric signed magnetic flux of the $\mathrm{CH}$, and from the part where the CME peels off ('dimming' area hereafter). The data used for this task are from HMI (Scherrer et al. 2012) onboard SDO (Pesnell et al. 2012). Data have been aligned and derotated.

The magnetic flux is computed with a threshold of $3 \sigma$ in HMI LOS-magnetograms, that is, about $45 \mathrm{G}$ in absolute value. Signed magnetic flux is computed for the coronal hole, in a box enclosing it with coordinates $\mathrm{x}=[-800,800], \mathrm{y}=[-350,-980]$ arcsec. The signed magnetic flux is also computed into a circle of radius equal to 125 arcsec centered at $\mathrm{x}=-24, \mathrm{y}=-472 \operatorname{arcsec}$ (in the de-rotated last image) equivalent to the (green) dashed circumference shown in Figure 1, that is, the photospheric area below the ejected CME and subsequent dimming. The circle encloses the polarity inversion line (PIL) crossing the area. The time range is from January 2 00:00 UT to January 3, 23:36 UT.

In Figure 2 (left), the positive and negative magnetic fluxes are displayed. Fluxes are mostly constant for the region of the coronal hole, with larger values for the negative flux, which is the main polarity of the $\mathrm{CH}$. In Figure 2 (right), fluxes are more variable 

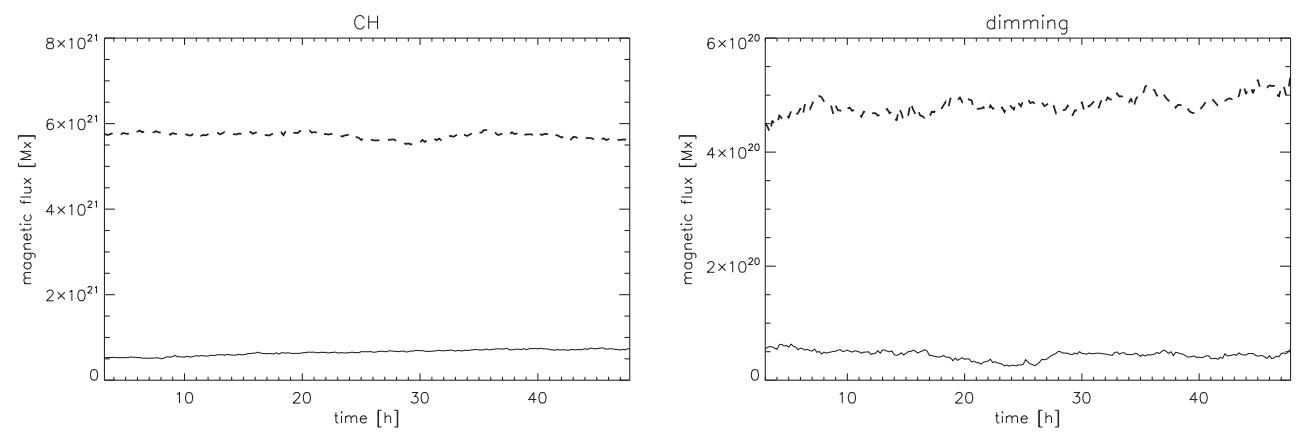

Figure 2. Left: positive and negative flux (thick, dashed line) of the whole CH. Right: positive and negative (thick, dashed line) flux of the dimming.
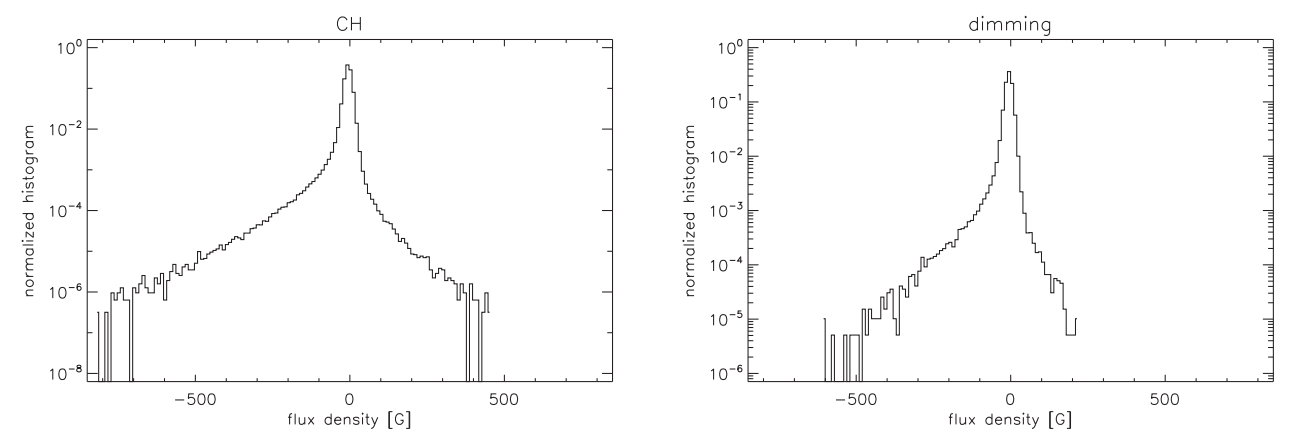

Figure 3. Left: Histogram of the signed magnetic field in the whole CH. Right: Histogram of the signed magnetic field of the dimming.

around the PIL, but the negative polarity still dominates. Although there are some flux increase and decrease with time, they are not apparent signatures of a flux rope formation or disappearance, nor any relevant change around $t=24 \mathrm{~h}$, when the dimming starts to appear.

The panels in Figure 3 display the histogram of flux density distributions in the last magnetogram, for the box and the circle. They are computed for the last magnetogram of the series and with a bin size of $10 \mathrm{G}$. It is evident that is highly skewed to the main polarity of the coronal hole, as suggested by Krista \& Gallagher (2009). The histogram of the dimming is more symmetric as positive and negative areas appear to be equivalent in area, since they are a part of the polarity inversion line.

\section{Discussion and conclusions}

We have checked whether the photospheric magnetic flux suffers any change at the $\mathrm{CH}$ total area and dimming area, due to the probable emergence/ejection and reconnection of the flux rope. However, it is not evident in Fig. 2, since no relevant polarity variation is observed in the dimming. The average magnetic flux in the $\mathrm{CH}$ is mainly constant during almost two days, with about $3 \%$ of the pixel area box with flux density values above the threshold. In Fig. 3, the large negative-polarity coronal hole magnetic density values are shown as a highly skewed distribution. The photospheric magnetic density corresponding to the dimming is more balanced in the histogram, as it corresponds to a PIL area.

Regarding spatial configurations and their possible consequences, the solar southern pole is oriented towards Earth in January. This empirical effect is maximum in March 
(in September, the northern pole is the most oriented), due to the configuration of the axes of the Sun and the Earth, creating an effective pointing that actually produces an statistical increase in geomagnetic storms in these months (known as Russell-McPherron effect (Bartels (1932), Russell \& McPherron (1973)). Therefore, this pointing can be an important factor in the subsequent geomagnetic storm, since the large coronal hole was located in the southern pole.

\section{Acknowledgements}

We thank HMI/SDO and Proba2 data and teams. SWAP is a project of the Centre Spatial de Liege and the Royal Observatory of Belgium funded by the Belgian Federal Science Policy Office (BELSPO). J. P. acknowledges funding from IAU to attend IAUS327 and UAH-travel grants. She also acknowledges funding from the Spanish MINECO project AYA2013-47735-P.

\section{References}

Bartels, J. 1932, Terrestrial Magnetism and Atmospheric Electricity, 37(1), 1-52.

Cid, C., Palacios, J., Saiz, E., \& Guerrero, A. 2016, ApJ, 828, 11.

Dasso, S., Mandrini, C. H., Démoulin, P., \& Luoni, M. L. 2006, A\&AA, 455, 349.

Halain, J.-P., Berghmans, D., Seaton, D. B., et al. 2013, Solar Phys., 286, 67.

Kataoka, R., Shiota, D., Kilpua, E., \& Keika, K. 2015, GRL, 42, 5155.

Krista L. \& Gallagher P., 2009, Solar Phys., 256, 87.

Lavraud, B., Ruffenach, A., Rouillard, A. P., et al. 2014, JGR, 119, 26.

Lugaz, N., Downs, C., Shibata, K., et al. 2011, ApJ, 738, 127.

Lugaz, N., Farrugia, C. J., Davies, J. A., et al. 2012, ApJ, 759, 68.

McComas, D. J., Gosling, J. T., Winterhalter, D., \& Smith, E. J. 1988, JGR, 93, 2519.

Pesnell, W. D., Thompson, B. J., \& Chamberlin, P. C., 2012, Solar Phys., 275, 3.

Ruffenach, A., Lavraud, B., Farrugia, C. J., et al. 2015, JGR, 120, 43.

Russell, C. T., and R. L. McPherron 1973, JGR, 78, 92.

Scherrer, P. H., Schou, J., Bush, R. I., et al., 2012, Solar Phys., 275, 207. 